\title{
Monitoring Outcome of Joint Arthroplasty in Italy: Implementation of the National Registry
}

\author{
Marina Torre ${ }^{1}$ Emilio Romanini ${ }^{2} \quad$ Gustavo Zanoli ${ }^{3} \quad$ Eugenio Carrani ${ }^{1} \quad$ Ilaria Luzi ${ }^{1}$ Luisa Leone ${ }^{4, \dagger}$ \\ Stefania Bellino ${ }^{5}$
}

${ }^{1}$ National Center for Clinical Excellence, Healthcare Quality \& Safety, Istituto Superiore di Sanità, Rome, Italy

${ }^{2}$ Casa di Cura San Feliciano, Rome, Italy

${ }^{3}$ Dipartimento di Ortopedia, Casa di Cura Santa Maria Maddalena, Occhiobello (RO), Italy

${ }^{4}$ National Center of Epidemiology, Surveillance and Health

Promotion, Istituto Superiore di Sanità, Rome, Italy

${ }^{5}$ Department of Infectious Diseases, Istituto Superiore di Sanità,

Rome, Italy

Joints 2017;5:70-78.

\begin{abstract}
Address for correspondence Marina Torre, MEng, National Center for Clinical Excellence, Healthcare Quality \& Safety, Istituto Superiore di Sanità, Viale Regina Elena, 299-00161, Rome, Italy (e-mail: marina.torre@iss.it).
\end{abstract}

\begin{abstract}
Keywords

- arthroplasty registry

- hip arthroplasty

- knee arthroplasty

- medical device

Purpose Arthroplasty registries have an important role in improving outcomes in joint surgery. As the demand for joint arthroplasty continues to increase, growing attention is being paid to the establishment of national registries, which contribute to the enhancement of the quality of patients' care. Indeed, providing postmarketing surveillance data in terms of safety and effectiveness of medical devices, registries contribute to the best orthopaedic practice and support public health decision making. In this context, a project aimed at implementing a national arthroplasty registry in Italy has appeared to be essential, and the activities performed in the last years have consolidated data collection of hip and knee replacements.

Methods Based on a close cooperation among public health institutions, clinicians, and involved stakeholders, the architecture of the registry is built on three pillars: (1) data collected using Hospital Discharge Records (HDRs) integrated by an additional dataset, (2) implants identified and characterized in a dedicated medical devices library, and (3) a federation of regional registries coordinated by a public health institution, the Italian National Institute of Health.

Results Besides the organizational structure, statistical analyses on joint arthroplasty from national HDR database (2001-2014) and Italian registry data (2014) are presented. Currently, the institutions participating in the registry on a voluntary basis show $80 \%$ of completeness for hip and $58 \%$ for knee, and represent approximately $18 \%$ of the national volume.

Conclusion To make data collection effective, participation should be mandatory and ruled by a national law.

Level of Study Level III, observational analytic study.
\end{abstract}

\section{Introduction}

The fast development of joint replacement technology with proliferation of new implant brands and types that largely

$\dagger^{\dagger}$ Luisa Leone passed away on October 16th, 2014. lack data on their long-term effectiveness requires an objective monitoring of the market, as failed innovation has already been highlighted in the past, ${ }^{1}$ and is still a critical issue. ${ }^{2}$ New devices are designed and manufactured with the expectation that they will be equivalent or superior to existing products, but unfortunately, this is not always 
true as reported by a study conducted by the Australian National Registry that showed a significantly worse outcome in $30 \%$ of new devices. ${ }^{3}$ In Scandinavia, national arthroplasty registries were established in the late 1970s to collect data on implanted devices using revision surgery as endpoint for failure, and these became a solid source of data for health researchers. ${ }^{4}$ Centralized data collection allowed rapid detection of worse performing products and easy recall of patients for clinical review; moreover, the participation in joint replacement data collection stimulated a continuous process of quality improvement through feedback from orthopaedic surgeons, manufacturers, and other stakeholders. ${ }^{5}$ Since then, data from the Scandinavian registries have become a reference standard for the evaluation of implant survival rates even outside the original boundaries and have stimulated the interest for organizing a national registry in many other countries. ${ }^{6}$ However, exporting results and methods in different geographic and socioeconomic contexts raises some critical issues that were not always properly considered in the past, leading to delay of some projects. ${ }^{7}$ A national data collection is mandatory to evaluate the national clinical practice and build a local reference, including implants not used in the countries where a registry is available, to stimulate a quality improvement process in the interest of patients, surgeons, and device manufacturers. ${ }^{8-10}$ Currently, several countries have national joint arthroplasty registries with a high level of completeness both in Europe and worldwide (http://www. isarhome.org/links). The European Parliament and the Council, in their proposal of regulation on medical devices, invited the Commission and the Member States to take all appropriate measures to encourage the establishment of registries for specific types of devices to gather postmarket experience and contribute to the independent evaluation of the longterm safety and performance of devices. ${ }^{11}$

In Italy, in 2006, the Directorate General of Medical Devices and the Pharmaceutical Service of the Ministry of Health $(\mathrm{MoH})$ charged the Italian National Institute of Health (Istituto Superiore di Sanità, ISS) for coordinating a project to establish a national joint registry, the Italian Arthroplasty Registry (Registro Italiano ArtroProtesi, RIAP).

The aim of this paper was to describe the organization of RIAP within the Italian health context as well as the operating tools and strategies developed for its realization, and to present and discuss the data on joint replacements collected by Hospital Discharge Records (HDRs) and by the Registry.

\section{Methods}

\section{The National Health System}

In Italy, the National Health System (NHS) is public. It was set up in 1978 (Law 23, December 1978, n. 833) and is based on three fundamental principles, namely universality of assistance, equality of access, and solidarity. Since then, fundamental changes have been done concerning the organizational structure of the NHS. The most important of them happened in 2001 stating that every region (and not the central govern- ment) is responsible for planning and organizing its own health services, including economic decisions, in a situation of absolute autonomy within the framework of the three principles mentioned above.

All the hospitalizations (ordinary and day-hospital admissions) made in all public and private Italian hospitals are routinely collected in the HDR database, a systematic data collection on health care at national level, which includes both demographics and clinical information, such as primary and secondary diagnosis, primary and secondary procedure, type of discharge, hospital length of stay, and hospitalization burden. Diagnoses and procedures are coded using the "International Classification of Diseases, 9th Revision, Clinical Modification" (ICD-9-CM). Data are sent by hospitals to the Regional Health Authority that before the transmission to the $\mathrm{MoH}$ is responsible of quality checking. Reimbursement of hospital admissions is provided by the region and computed using Diagnosis Related Groups on the reported main diagnosis. Reimbursements are based on rules established by each region; so, they can differ for the same procedure throughout the country.

\section{Medical Devices Regulation}

The 2003 Government Financial Law foresaw the realization of the General Repository (GR) of the medical devices (MDs). It includes all the MDs marketed in Italy and used within the NHS, and is constantly updated by the manufacturers. The recorded devices are classified in homogeneous classes following the National Classification of Medical Devices (CND) developed by the Unique Commission on Medical Devices and published in a $\mathrm{MoH}$ decree.

\section{The Registry Project}

Since 2000, three Italian regions independently started their regional registry. ${ }^{12-14}$ Meantime, given the patients' freedom to move across regions to get assisted by different healthcare providers, a high interregional mobility was observed, which implies data loss for those patients operated and "registered" in one region and then revised in a different one. ${ }^{15,16}$ The high interregional migration rate and the need to monitor the high number of implanted devices were the main issues boosting the need for a national registry. Therefore, in 2003, an initiative was started by ISS for networking regions, orthopaedic scientific societies, and $\mathrm{MoH}^{13}$ In this context, in 2006, the Medical Devices and Pharmaceutical Service General Directorate appointed ISS to lead a national project aimed at implementing a national arthroplasty registry, organized as a federation of regional registries. A Steering Committee was instituted, with representatives from all the involved stakeholders, including public health institutions, scientific societies, biomedical industries, and patients' associations. The model for data collection and MD traceability was designed, tested in the already existing regional registries, and extended to the other regions progressively enrolled on a voluntary basis.

The RIAP project is a research line of ISS; its activities are performed by a multidisciplinary working group consisting of bioengineers, statisticians, epidemiologists, and 
information technology experts in force at ISS. Surgeons designated by the Italian Society of Orthopaedics and Traumatology and by the involved regions, voluntary cooperate to the project as external consultants. Other stakeholders involved are the National Association of Medical Device Manufacturers, Assobiomedica, and the National Association of People affected by Rheumatic Diseases. All the involved stakeholders, including public health institutions, scientific societies, biomedical industries, and patients' associations are represented in the Steering Committee.

\section{RIAP Data Collection}

The data collection flow uses HDR integrated by an additional minimum dataset (MDS), including patients' characteristics, surgical procedures, and information to identify the implanted devices. MDS was developed by a thorough analysis and comparison of the data collection sheets used by the already existing three regional registries. The list of information to be collected, defined for hip and further adapted for knee replacements, include hospitalization code, joint, operated side, type of procedure, diagnosis for primary intervention and revision, previous procedure, approach, fixation, CND code, manufacturer, and device catalog code. Particular attention was paid to the punctual identification of every component of the implant. To this aim, a dedicated library (RIAP MD Library), including catalog code, manufacturer's name, description, CND code, and GR registration code
(MD-GR ID), was implemented and continuously fed by manufacturers. Currently, the library includes more than 55,000 catalog numbers of 60 manufacturers available through the RiDi Webservice. Thus, the data collection model consists of two flows, the clinical data collection (green) and the MD identification and characterization (blue) ( - Fig. 1 ).

Since 2007, regions have been progressively enrolled into the project. Currently, data collection actively involves 10 regions of the 11 enrolled and 2 autonomous provinces. Three regions started collecting data in 2015 and two have just been enrolled in 2016 (-Fig. 2). In 2014, six regions and a high-volume hospital of Liguria collected data on hip and knee replacements and two regions collected data only on hip replacements. To respect the regional autonomy, several different ways of implementing the common protocol of data collection were accepted. Regions having an already established regional arthroplasty registry and regions that integrated MDS into their local HDR flow directly extract RIAP data from their existing databases. For the other participating regions, a web application (RaDaR) was developed by ISS to facilitate surgeons in collecting MDS. All the regional coordinating centers are responsible of the linkage between HDR and MDS. The linked data are sent to RIAP through the SOnAR web application (-Fig. 1). ISS accesses all data, whereas every participant can access only its own database. RIAP built an infrastructure to collect and analyze essential data, and return them in appropriate form, thus

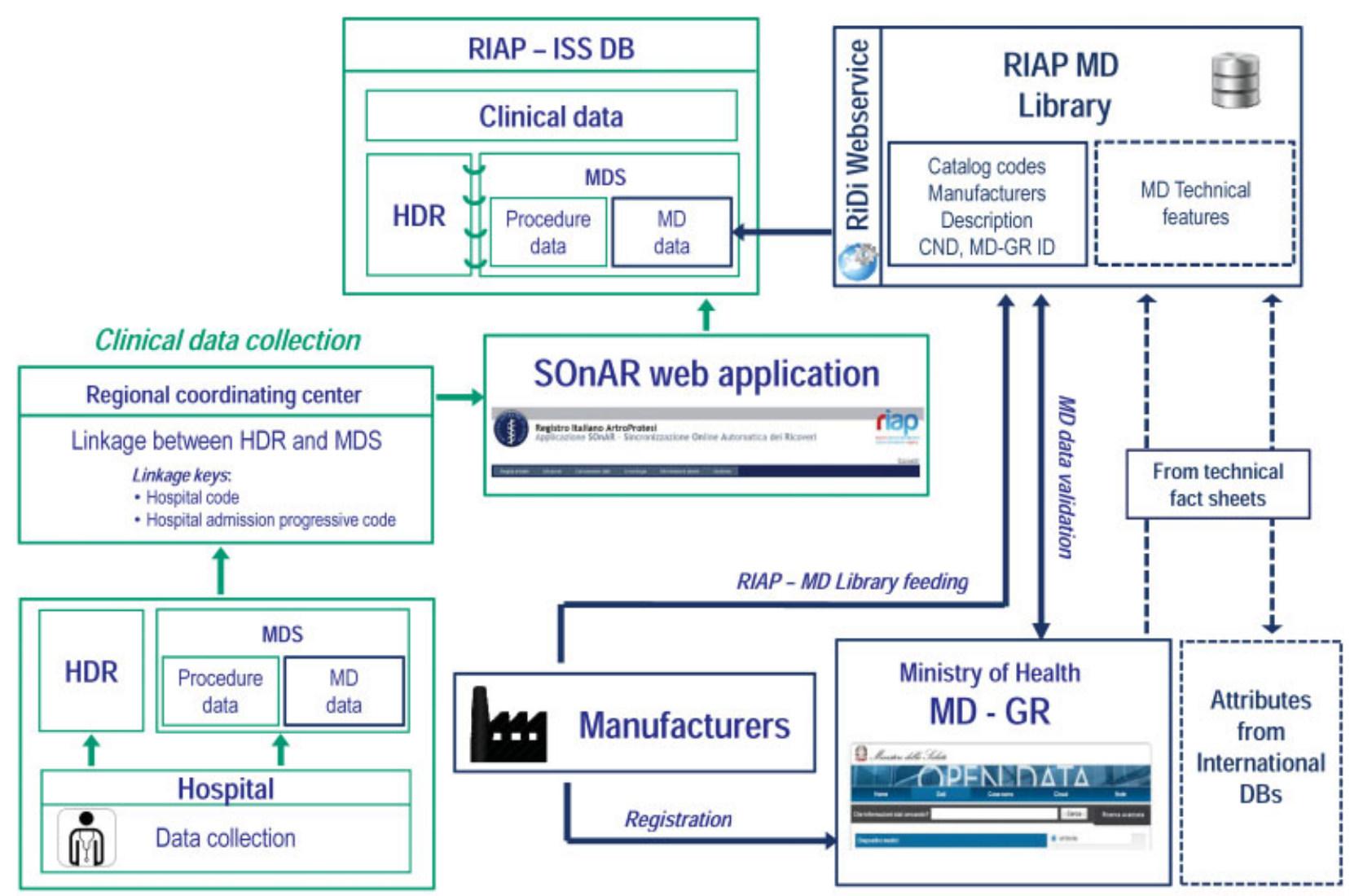

MD identification and characterization

Fig. 1 Italian Arthroplasty Registry. Flow diagram supporting the clinical data collection and the medical device identification and characterization. 

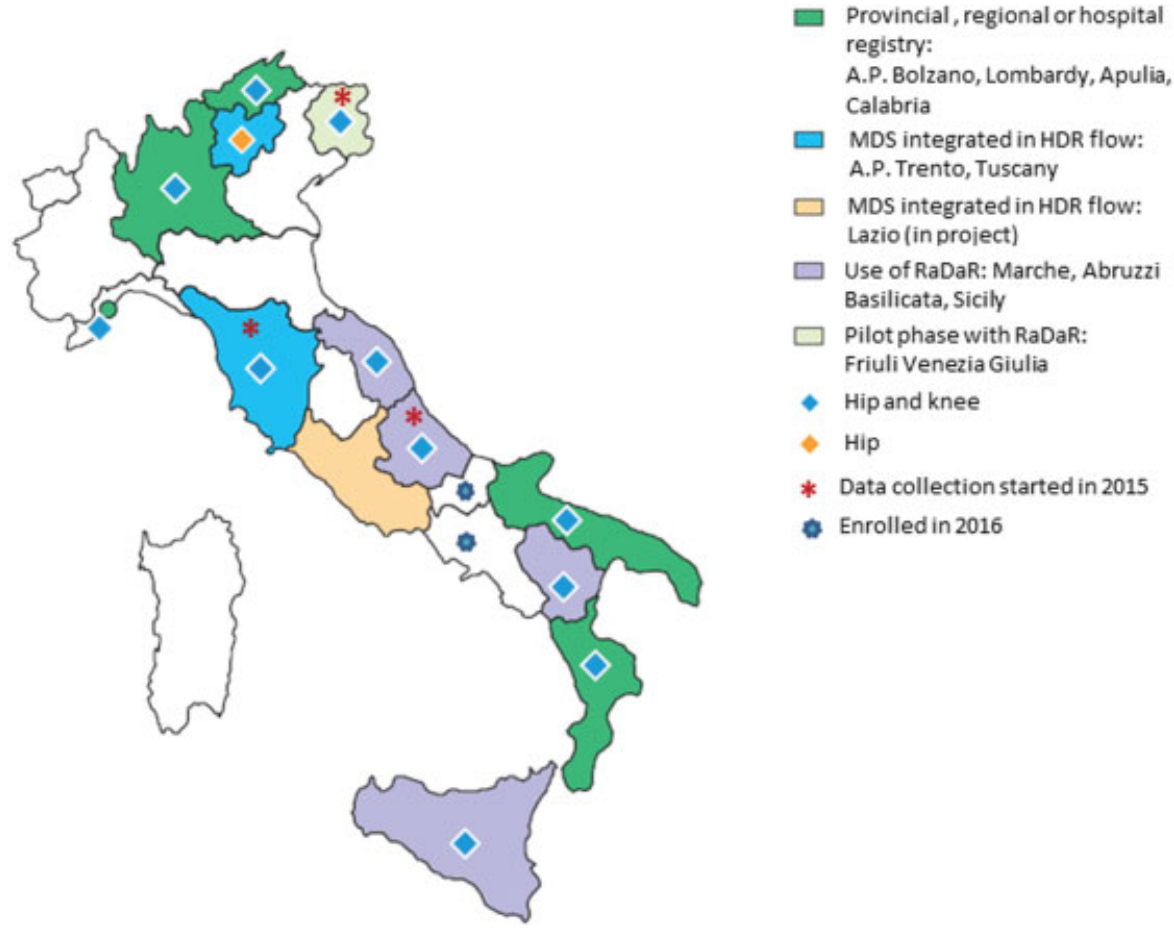

Fig. 2 Italian regions, autonomous provinces, and institutions currently enrolled in the Italian arthroplasty registry and procedure adopted for data collection.

providing useful information not only to physicians and orthopaedic surgeons on primary joint replacements, surgical technique, and implanted prostheses, but also to manufacturers to strengthen postmarket surveillance. Moreover, manufacturers feeding MD Library receive a feedback on quality control about medical device identification data sent to RIAP.

Until now, three national reports have been published; the first describes the project development and the methodological approach, the second is focused on data collection implementation, and the third is based on the necessity to improve the data quality, essential for enhancing the reliability of the analyses and the rightness of observations that can be drawn (http://www.iss.it/riap).

\section{Informed Consent and Treatment of Personal Data}

Patients enrolled into the registry are requested to sign the informed consent form after reading patient's information sheet, including the purpose of the study, type of recorded data, and health benefits of participation. Following the provisions of the law on protection of personal data (Legislative Decree n. 196/2003), information is recorded in anonymous form and used only for scientific research projects. Indeed, subject is attributed an identification code and patient's identification data are never transmitted to the registry; connection between them and the code is allowed only to hospital staff for justified reasons such as data quality control or patient recall. The collection and use of personal data occur in a strictly confidential manner through computer systems and data are protected by an access-controlled system that meets the Italian law requirements. Patient's information sheet and informed consent form were approved by the Ethics Committee of the ISS.

\section{Data Analysis}

Data analysis was performed on both the national HDR database, which includes all joint arthroplasties performed in public and private hospitals, and the data collected by RIAP, the Italian registry database, which uses HDR data integrated by additional information essential to describe the procedures and identify the implants. The first analysis was based on admissions that occurred from 2001 to 2014 and provided a general overview of joint arthroplasties throughout the country by mapping volume of activity. Analysis on the RIAP database referred to the data collected in 2014 by the hospitals of five regions, two autonomous provinces and one public foundation participating in the project. Statistical analysis was performed using SAS software, version 9.3 (SAS Institute, Cary, North Carolina, United States) and STATA, version 11 (Stata Corporation, College Station, Texas, United States).

\section{Results}

\section{Hip Arthroplasties}

The number of primary total hip replacements performed in the Italian hospitals from 2001 to 2014 increased steadily. In particular, elective replacements increased by $44.5 \%$, from 39,144 in 2001 to 56,561 in 2014, with an annual growth rate of $2.9 \%$, while the percentage of hip replacements with diagnosis of fracture of neck of femur (ICD-9-CM code, 820) increased to a lesser extent $(42.7 \%)$ ( - Fig. 3A). Also, hip 

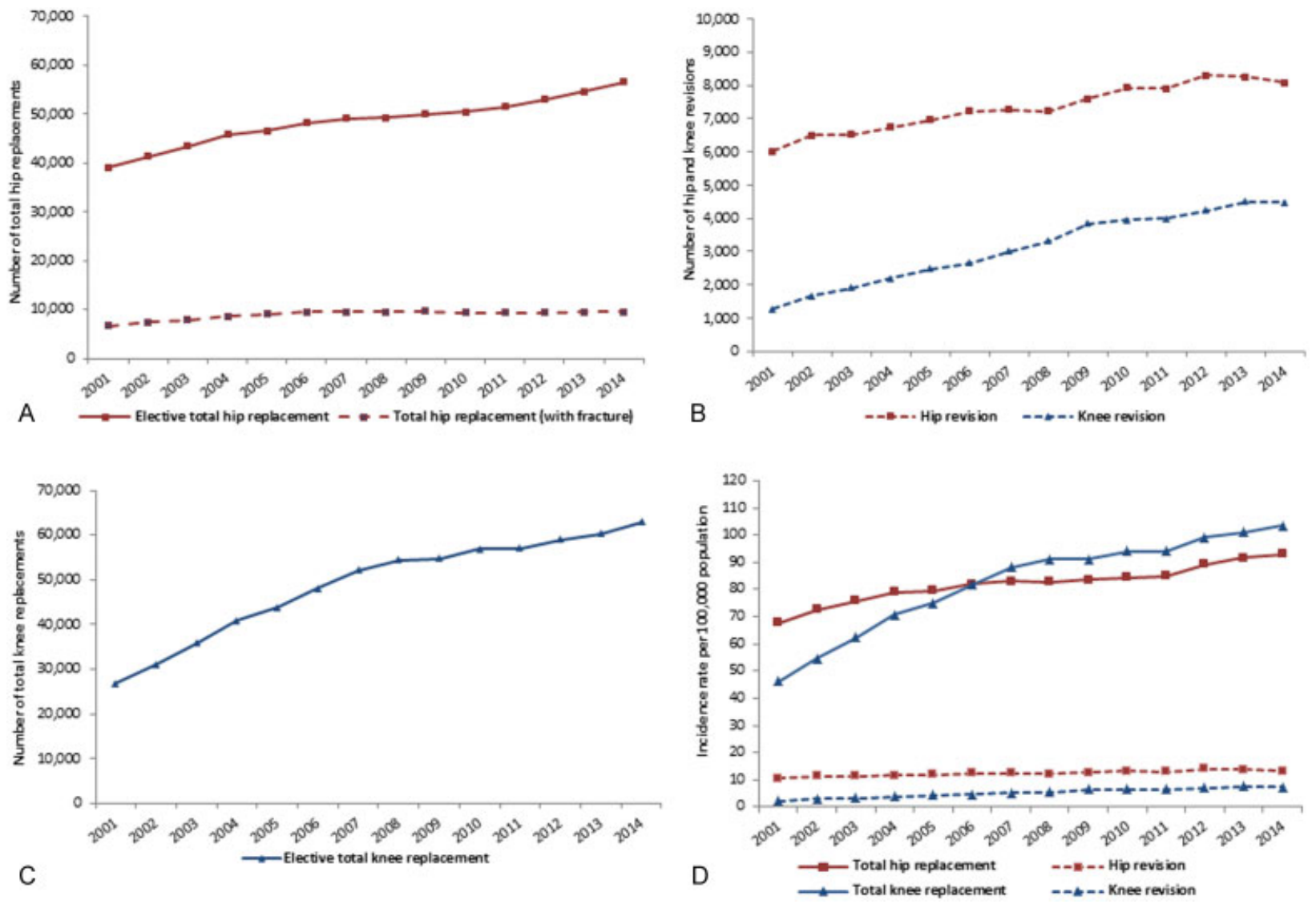

Fig. 3 Absolute numbers and incidence rates of hip and knee arthroplasties from 2001 to 2014 in Italy. (A) Primary total hip replacement (with or without fracture). (B) Hip and knee revision. (C) Primary total knee replacement. (D) Incidence rates of hip and knee arthroplasties, both primary and revisions. Data source: National Hospital Discharge Records (HDRs) database.

revisions increased over time, from 6,015 in 2001 to 8,069 in 2014 (34.1\%), with an annual growth rate of 2.3\% (- Fig. 3B).

In $2014,91,428$ primary replacements and 8,069 revisions were performed: $66.4 \%$ and $25.4 \%$ procedures were detected for total and partial replacements, respectively, whereas, $0.1 \%$ procedures were performed for resurfacing. Most of the total hip replacements were due to osteoarthritis (80.9\%), followed by femoral neck fracture (14.1\%).

Despite a low national completeness, RIAP data reflected the Italian distribution. Indeed, $66.8 \%$ total replacements, $27.5 \%$ partial replacements, and 5.7\% revisions were recorded. In particular, 14,724 were elective total replacements, 1,749 were due to a fracture, and 1,410 were revisions. The mean age of patients undergoing elective total hip replacement was 68 years and 55\% of surgeries were performed in females. The most frequent diagnoses in elective total replacement were primary osteoarthritis (87.6\%), avascular necrosis of femoral head (4.7\%), and dysplasia/ congenital dislocation (4.1\%, - Fig. 4A). Aseptic loosening (acetabular, femoral or both; $41.5 \%$ ), dislocation (16.2\%), and periprosthetic fracture $(10.8 \%)$ were the most common indications for revision surgery (-Fig. 4B). Patients were mainly operated on the right side, $55.0 \%$ for primary intervention and $50.3 \%$ for revision ( $\mathbf{- F i g . ~} \mathbf{5 A}$ ); the most used surgical approach was posterolateral, followed by lateral for both primary and revision arthroplasties (-Fig. 5B). Fixation of MDs was cementless for $92.5 \%$ of interventions (- Fig. 5C). Concerning the characteristics of implants, the most common articular bearing surface was ceramic-on-polyethylene (52.9\%), followed by ceramic-on-ceramic (23.5\%), metal-onpolyethylene $(21.3 \%)$, ceramic-on-metal $(0.9 \%)$, metal-onmetal $(0.8 \%)$, and metal-on-ceramic $(0.6 \%)$.

The analysis of the distribution of the Italian hospitals per volume of activity showed that $45 \%$ of hospitals performed low volumes ( $\leq 50$ operations per year), particularly concentrated in the southern regions ( $\mathbf{- F i g . 6 A}$ ).

\section{Knee Arthroplasties}

The number of knee arthroplasties increased sharply over the last 13 years, from 26,787 in 2001 to 62,886 in 2014 (134.8\%) for primary replacements (-Fig. 3C), with an annual growth rate of $6.8 \%$, and from 1,269 in 2001 to 4,479 in 2014 (253.0\%) for revisions (-Fig. 3B), with an annual growth rate of $10.2 \%$. The revision burden slightly increased over time, from 4.5 in 2001 to $6.6 \%$ in 2014 . The annual incidence rate particularly increased for primary total knee replacements that doubled, from 46.3 to 103.5 per 100,000 population, overcoming that of hip arthroplasties in 2006 (-Fig. 3D). In 2014, 62,886 primary replacements and 4,479 revisions were performed. Primary arthroplasties were performed almost exclusively for osteoarthritis (96.9\%). 

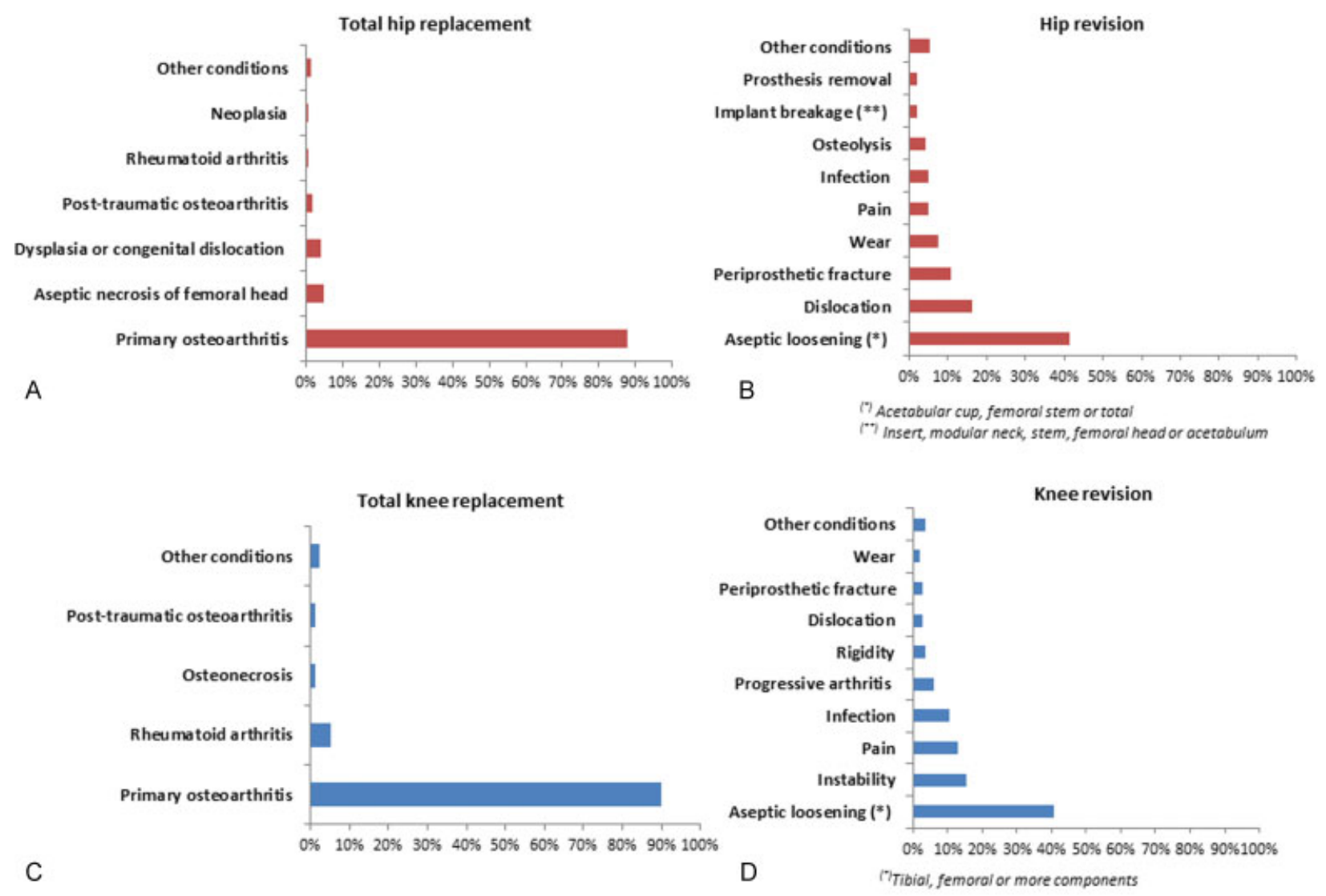

Fig. 4 Elective total replacement and revision by diagnosis. (A-B) Hip. (C-D) Knee. Data source: Italian Arthroplasty Registry database, 2014.

Even in this case, data collected by RIAP reflected the national distribution with $95.7 \%$ primary total replacements $(4,726)$ and $4.3 \%$ (212) revisions. The mean age of patients undergoing primary replacement was 71 years, and $70 \%$ of procedures were performed in females. The most frequent diagnoses were osteoarthritis (90.1\%), rheumatoid arthritis (5.2\%), and osteonecrosis (1.1\%) for primary replacement (-Fig. 4C). Aseptic loosening of tibial, femoral, or more components (40.7\%), instability (15.5\%), and pain (12.9\%) were the most common indications for revision surgery (-Fig. 4D).

More than $70 \%$ of patients were operated on the right side ( - Fig. 5D), while the most used surgical approach (>90\%) was medial parapatellar (-Fig. 5E). Fixation of MDs was cemented for more than $93 \%$ of interventions (-Fig. 5F).

Also, in this case, more than half of the Italian hospitals (54\%) performed low volume of activity, particularly concentrated in the southern regions (-Fig. 6B). This scenario was expected, because in the previous years, a greater concentration of knee arthroplasties was performed in the central-northern regions.

\section{Discussion}

Arthroplasty registries have shown to be a reliable source of information, providing postmarketing monitoring of devices and improving orthopaedic practice worldwide. ${ }^{17}$

In Italy, the registry was developed as federal, thereby respecting the regional autonomy. This means that every region is responsible in selecting the most effective way to implement the data collection in its local context, if all the data requested by the national protocol are regularly delivered to the ISS. Moreover, it is based on a solid network involving all the stakeholders, and the ISS, in its role of reference center, offers the opportunity of sharing knowledge and experience, thereby providing benefits to the whole project. Following this approach, some regions included the additional dataset in their local information flows, incorporating the registry data collection in their regional health system. Moreover, given the unavailability of a Unique Device Identifier (UDI), which should ensure a complete traceability of the implant in case of a product recall, the cooperation with the manufacturers to implement the RIAP MD Library integrated into the registry data collection is crucial. As a future perspective, the development of a UDI system harmonized at the European level ${ }^{18}$ will be the best way to ensure effective traceability of MDs in the EU and will support the interoperability of different systems to monitor the use of MDs both at national and international levels.

Completeness and correctness of information included in the registry database are an important issue. Quality control procedures to ensure that the collected data fit with the record layout and meet a set of specific metrics are in development. In 2014, the quality rate (the ratio between the number of records considered in the analysis and those registered in the database) was $95 \%$ and $89 \%$ for hip and knee replacements, respectively. The total number of 


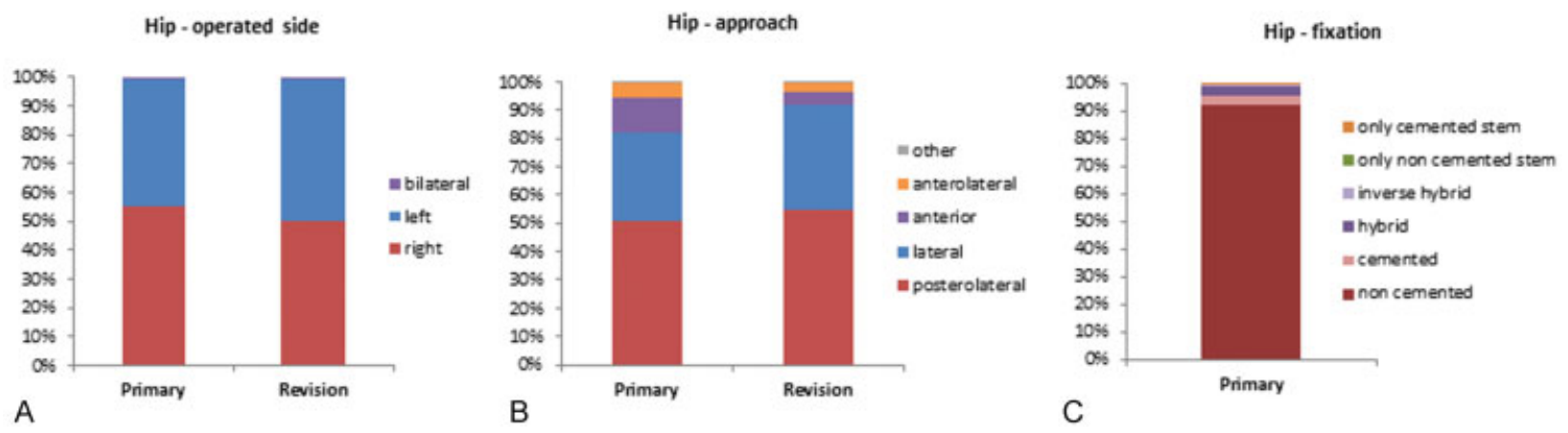

Revision: total or partial revision, removal of prosthesis, conversion from partial to total replacement, spacer revision Hybrid: cementess cup and cemented stem Reverse hybrid: cemented cup and cemendess stem
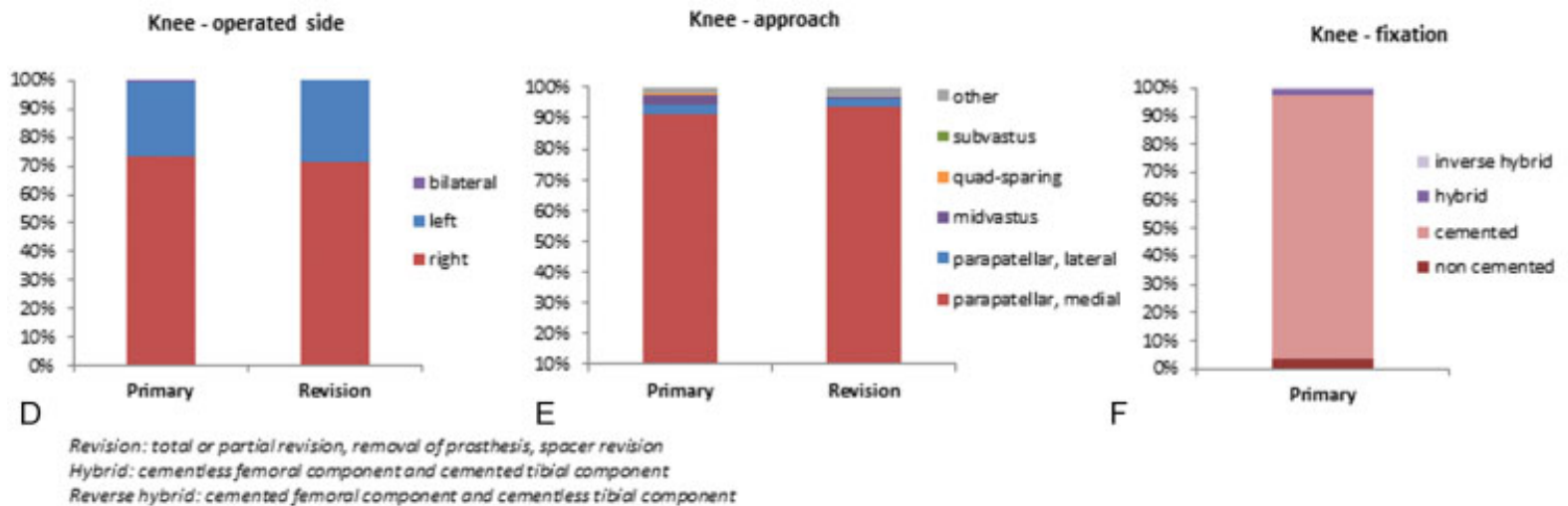

Hybrid: cementiess femord component and cemented tibial componen: Reverse hybrid: cemented femoral component and cementiess tibial component

Fig. 5 Characteristics of surgical practice for elective total replacement and revision procedures in 2014. (A-C) Hip. (D-F) Knee. Data source: Italian Arthroplasty Registry database, 2014.

procedures recorded in 2014 covered approximately $80 \%$ of hip and $58 \%$ of knee arthroplasties performed in the regions involved in the data collection. Adherence to the protocol varied among regions, from $15 \%$ to $100 \%$ for hip and from 18 to $100 \%$ for knee; the highest values were reached in the regions where the registry was instituted by law and participation was mandatory or where a promotion of the importance of data collection and motivation of surgeons was made by the regional government. Data collected in 2014 represented nearly $18 \%$ of the national volume of
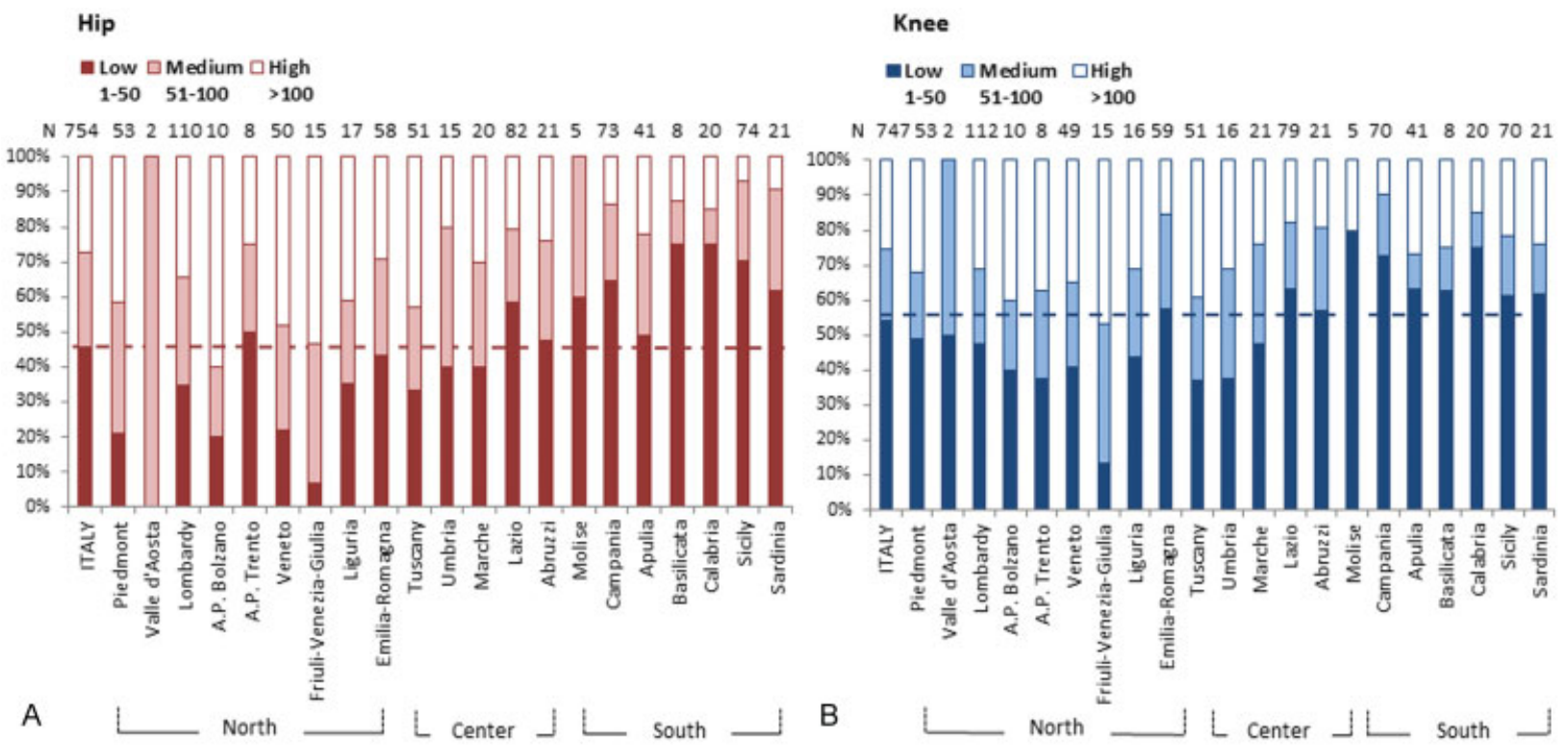

Fig. 6 Percentage of hospitals by volume of activity (number of operations per year) by Italian regions. (A) Hip. (B) Knee total replacements. The dotted line represents the national level of low volume of activity. Data source: National Hospital Discharge Records (HDRs) database, 2014. 
activity, 25\% for hip and 7\% for knee replacements; however, it still lacked the participation of four regions with the highest volumes.

To improve the quality of the collected data, audits are going to be planned to ensure that the information of the HDRs are correctly reflected into the registry. In addition to the already collected data, including measures on Patient Reported Outcomes (PROs) would enhance completeness and significance of data, thereby helping providers to improve quality of life of patients after joint replacement surgery. To this aim, RIAP working group validated the Italian version of the Hip disability and Osteoarthritis Outcome Score (HOOS) questionnaire by a longitudinal study on one hundred forty-five patients enrolled in five hospitals throughout the country and followed for 6 months. In the first phase, PROs data could be collected from a sample of hospitals participating in RIAP based on specific research projects, and only at a later time point, to extend the survey.

To be effective, a registry should include more than $90 \%$ of actually performed procedures. ${ }^{19}$ As demonstrated by the Canadian Joint Replacement Registry, ${ }^{20}$ to achieve this goal, the participation should be mandatory. In Italy, the national registry is still based on voluntary participation. Since its inception, the project has been widely disseminated within the scientific community by participating in the national and regional orthopaedic congresses to stimulate surgeons' participation and make them aware of the importance and the benefits related to the implementation of a national registry. Contacts have been established by the RIAP coordinator with the heads of the regional administrations, and specific technical meetings have been organized involving regional body's officials responsible for the data collection to support the implementation of the registry.

In 2012, the National Governmental Law instituting the registry of MDs for epidemiological surveillance (Law 221/ 2012 ) was approved. However, the process for its coming into force requires the application of a further law-decree that has been just approved (Decree of the President of the Council of Ministers of March 3, 2017).

As showed by the national HDR data analysis, in Italy, the number of joint arthroplasties progressively increased in the last 13 years. This is in line with a systematic review of the literature based on 24 studies performed in different countries, which found that the utilization rates for total hip and knee arthroplasty increased over the last 2 to 3 decades. $^{21}$ With aging of the population and increased longevity, the joint replacements are projected to increase even further, although the rates vary by country due to differences in socioeconomic status, health care delivery systems, patient preferences, and prevalence of osteoarthritis, the most common underlying cause for this type of intervention. ${ }^{21}$ The increase in the number of arthroplasties raises some critical issues directly connected to health policies. In fact, if the projections available for the United States ${ }^{22,23}$ and Sweden ${ }^{24}$ are applied to our country, the current context of insufficient and further expected decrease of orthopaedic surgeons will probably generate an important unbalance between demand and offer of these kind of procedures. ${ }^{25}$
Improving quality and effectiveness of health care is one of the priorities of health policies. Hospital volume represents a measurable variable with a relevant impact on effectiveness of health care. Therefore, there is a need for an accurate evaluation of the available scientific evidence to identify qualitative and quantitative standards of hospital care. Evidence of a positive association between volume of care and outcome was demonstrated for different clinical areas, including hip and knee arthroplasty. ${ }^{26-28}$ In Italy, the proportion of hospitals with a yearly low volume of activity ( $\leq 50$ procedures) is quite high, $45 \%$ and $54 \%$ for hip and knee primary replacements, respectively. This highlights the need that strategies aimed at concentrating these kinds of procedures in medium-to-high volume hospitals should be adopted by the central and regional governments.

Overall, hip and knee replacements were performed with large distribution all over the country; however, referral to hospitals far from the area of residency is still common with massive interregional mobility. Patient interregional mobility continues to be significant and the northern Italian regions are more attractive to the users. ${ }^{15,16}$

Although the national completeness of the arthroplasty registry is still low, characteristics of surgical practice and clinical conditions for hip and knee interventions from RIAP study are quite in line with data of other registries (http:// www.isarhome.org/links).

Arthroplasty registry experience has shown that through the registries, it is possible to evaluate outcomes of total joint replacement providing information on implants survivorship and identifying previously unknown associated risk factors. ${ }^{17,29,30}$ Registry postmarket surveillance has proven to be a powerful method for detection of increased risk of implant failure. Moreover, in compliance with the industry and the orthopaedic community, registries should ensure that a more cautious approach is used when new technologies are introduced. ${ }^{17}$ In this context, together with observational studies and clinical trials, the registries are reliable instruments which can provide policy and decision-makers with objective evaluations, useful to address safe, effective and patient-focused health policies.

Findings included in this article are a valuable snapshot of the Italian experience in implementing the national arthroplasty registry data collection, providing an information platform that will allow documentation on safety and effectiveness in the performance of these procedures over time. However, current results remain mainly descriptive pending high national completeness, long-term follow-up, and survival data on implants. Only the institution of the registry by law will allow to reach high level of completeness and its integration into the health care system will enhance its capacity to bring beneficial effects, influencing the decision makers at all levels.

\section{Funding}

The RIAP project is funded by the Italian Ministry of Health, Medical Devices and Pharmaceutical Service General Directorate. Further information concerning the project are available at www.iss.it/riap. 


\section{Acknowledgments}

We are most grateful to Marcella Marletta (Director of the Medical Devices and Pharmaceutical Service General Directorate of the Italian Ministry of Health) for supporting the project. We thank all the members of the present Steering Committee and of the past ones for their cooperation in the definition of the additional minimum dataset and the locally implementation of the data collection, and the Italian Arthroplasty Registry group including all the surgeons and health professionals actively participating in the Registry (a detailed list of the members of the Steering Committee and of the Italian Arthroplasty Registry group is available in the project website www.iss. it/riap). We are most grateful to Gabriele Tucci, Martina Del Manso, Annamaria Donato, and Rosaria Boldrini for their indispensable advice and cooperation. We would also like to thank Antonio Sette, Corrado Di Benedetto, and Mariano Santaquilani for the design and implementation of the informatics procedures supporting the data collection; Mascia Masciocchi, Simona De Santis, Gabriella Martelli, Stefano D'Ottavi, and Stefania Ceccarelli for their assistance in the technical and administrative management of the project, Lorenzo Fantozzi for his contribution to the figures preparation.

\section{References}

1 Huiskes R. Failed innovation in total hip replacement. Diagnosis and proposals for a cure. Acta Orthop Scand 1993;64(06): 699-716

2 Harris WH. Last decade in THA: unsettling and disappointing. J Arthroplasty 2014;29(03):648. Doi: 10.1016/j.arth.2013.10.011

3 Anand R, Graves SE, de Steiger RN, et al. What is the benefit of introducing new hip and knee prostheses? J Bone Joint Surg Am 2011;93(Suppl 3):51-54

4 Havelin LI, Fenstad AM, Salomonsson R, et al. The Nordic Arthroplasty Register Association: a unique collaboration between 3 national hip arthroplasty registries with 280,201 THRs. Acta Orthop 2009;80(04):393-401

5 Herberts P, Malchau H. Long-term registration has improved the quality of hip replacement: a review of the Swedish THR Register comparing 160,000 cases. Acta Orthop Scand 2000;71(02): 111-121

6 Serra-Sutton V, Allepuz A, Espallargues M, Labek G, Pons JM. Arthroplasty registers: a review of international experiences. Int J Technol Assess Health Care 2009;25(01):63-72

7 Niethard FU, Weise K. The German endoprosthesis register - a never-ending story?[in German] Z Orthop Unfall 2009;147(02): 149-150

8 Maloney WJ. The role of orthopaedic device registries in improving patient outcomes. J Bone Joint Surg Am 2011;93(24): 2241. Doi: $10.2106 / \mathrm{JBJS} .9323$ edit

9 Graves SE. The value of arthroplasty registry data. Acta Orthop 2010;81(01):8-9

10 Horan FT. Joint registries. J Bone Joint Surg Br 2010;92(06): 749-750

11 Regulation of the European Parliament and of the Council on medical devices, and amending Directive 2001/83/EC, Regulation (EC) No 178/2002 and Regulation (EC) No 1223/2009. Brussels, June 27, 2016
12 Toni A, Stea S, Bordini B, Traina F. Lost to follow-up in a hip prosthesis register. Experience of R.I.P.O. Acta Orthop Scand Suppl 2002;73(305):49-53

13 Germinario C, Torre M, Palmieri S, Lo Palco PL, Prato R. Regione Puglia. Registro regionale delle protesi d'anca [Apulia Region. The hip arthroplasty regional registry]. In: Progetto per l'istituzione del Registro nazionale degli interventi di protesi di anca [Design of the Italian National Hip Arthroplasty Register]. Torre M (Ed). Roma: Istituto Superiore di Sanità; 2005. (Rapporti ISTISAN 05/18)

14 Zagra L. Regione Lombardia. Registro Ortopedico Protesico Lombardo [Lombardy Region. The Orthopaedic Registry of Lombardy]. In: Progetto per l'istituzione del Registro nazionale degli interventi di protesi di anca [Design of the Italian National Hip Arthroplasty Register]. Torre M (Ed). Roma: Istituto Superiore di Sanità; 2005. (Rapporti ISTISAN 05/18)

15 Romanini E, Torre M, Manno V, Baglio G, Conti S. Chirurgia protesica dell'anca: la mobilità interregionale [Total hip replacement: the interregional mobility]. Giornale italiano di ortopedia e traumatologia. Ital J Orthop Traumatol 2008;34:129-134

16 Romanini E, Manno V, Conti S, et al. Mobilità interregionale e chirurgia protesica del ginocchio [Interregional mobility and total knee arthroplasty]. Annali di Igiene. Ann Hyg 2009;21(04):329-366

17 Malchau H, Graves SE, Porter M, Harris WH, Troelsen A. The next critical role of orthopedic registries. Acta Orthop 2015;86(01):3-4

18 European Commission: Commission Recommendation 2013/172/ EU of 5 April 2013 on a common framework for a unique device identification system of medical devices in the Union (Text with EEA relevance). OJEU 2013, L 99/17

19 Kolling C, Simmen BR, Labek G, Goldhahn J. Key factors for a successful National Arthroplasty Register. J Bone Joint Surg Br 2007;89(12):1567-1573

20 Hip and Knee Replacements in Canada. Canadian Joint Replacement Registry 2015 Annual Report. Available at: https://secure. cihi.ca/free_products/CJRR_2015_Annual_Report_EN.pdf

21 Singh JA. Epidemiology of knee and hip arthroplasty: a systematic review. Open Orthop J 2011;5:80-85

22 Badley EM, Davis AM. Meeting the challenge of the ageing of the population: issues in access to specialist care for arthritis. Best Pract Res Clin Rheumatol 2012;26(05):599-609

23 Kurtz S, Ong K, Lau E, Mowat F, Halpern M. Projections of primary and revision hip and knee arthroplasty in the United States from 2005 to 2030. J Bone Joint Surg Am 2007;89(04):780-785

24 Nemes S, Rolfson O, W-Dahl A, et al. Historical view and future demand for knee arthroplasty in Sweden. Acta Orthop 2015; 86(04):426-431

25 Torre M, Tucci G, Luzi I, Del Manso M. Joint arthroplasty in high functional demanding patients: epidemiologic data. Giornale Italiano di Ortopedia e Traumatologia 2015;41(Suppl 1):S1-S8

26 Amato L, Colais P, Davoli M, et al. Volume and health outcomes: evidence from systematic reviews and from evaluation of Italian hospital data [in Italian]. Epidemiol Prev 2013;37(2-3, Suppl 2):1-100

27 Glassou EN, Hansen TB, Mäkelä K, et al. Association between hospital procedure volume and risk of revision after total hip arthroplasty: a population-based study within the Nordic $\mathrm{Ar}$ throplasty Register Association database. Osteoarthritis Cartilage 2016;24(03):419-426

28 Singh JA, Ramachandran R. Does hospital volume predict outcomes and complications after total shoulder arthroplasty in the US? Arthritis Care Res (Hoboken) 2015;67(06):885-890

29 Inacio MC, Paxton EW, Dillon MT. Understanding orthopaedic registry studies: a comparison with clinical studies. J Bone Joint Surg Am 2016;98(01):e3. Doi: 10.2106/JBJS.N.01332

30 Lacny S, Bohm E, Hawker G, Powell J, Marshall DA. Assessing the comparability of hip arthroplasty registries in order to improve the recording and monitoring of outcome. Bone Joint J 2016; 98-B(04):442-451 